\title{
Pharmacokinetics of toltrazuril and its metabolites toltrazuril sulphoxide and toltrazuril sulphone in pregnant and non-pregnant goats
}

\author{
Sara T. Elazab ${ }^{1}$, Nahla S. Elshater ${ }^{2}$, Ahmed E. Elweza $^{3}$ \\ ${ }^{1}$ Mansoura University, Faculty of Veterinary Medicine, Department of Pharmacology, Mansoura, Egypt \\ ${ }^{2}$ Agriculture Research Center, Animal Health Research Institute, Giza-Dokki, Egypt \\ ${ }^{3}$ University of Sadat City, Faculty of Veterinary Medicine, Department of Theriogenology, Sadat City, Menoufia, Egypt
}

Received August 9, 2021

Accepted November 9, 2021

\begin{abstract}
The pharmacokinetic characteristics of toltrazuril (TZR) and its metabolites toltrazuril sulphoxide (TZR.SO) and toltrazuril sulphone (TZR.SO $)$ were assessed in non-pregnant and pregnant goats. Ten healthy Baladi female goats were allocated into two groups $(n=5$ per group): non-pregnant goats (group 1) and pregnant goats at 2-3 months of gestation (group 2). Toltrazuril was administered once orally to all goats at $20 \mathrm{mg} / \mathrm{kg}$. Plasma samples were collected at 0 (before TZR administration), 0.5, 1, 2, 4, 6, 8, 12, 16, 24, 48, $72 \mathrm{~h}$ and 5, 7, 9, 12, 16, 20, 24, 27, 30, and 35 days post therapy to measure the concentrations of TZR and its metabolites. In pregnant goats, the maximum plasma concentration $\left(\mathrm{C}_{\max }\right.$, time to reach $\mathrm{C}_{\max }\left(\mathrm{T}_{\max }\right)$, and the area under the plasma concentration-time curve from time zero to the last sample $\left(\mathrm{AUC}_{0 \text {-last }}\right.$ ) of TZR were significantly higher $(P<0.05)$ compared to the non-pregnant ones, whereas the volume of distribution (Vz_F_obs) and clearance $(\mathrm{Cl}$ _F_obs) were significantly lower $(P<0.05)$ in pregnant goats. No significant differences were observed in the elimination half-life $\left(\mathrm{T}_{1 / 2 \lambda}\right)$, and mean residence time (MRT) between the two groups. In non-pregnant goats, TZR.SO and TZR.SO could be detected in plasma until 12 and 30 days, respectively; whereas in pregnant goats, they were quantified up to 16 and 35 days, respectively. Conclusively, TZR was well absorbed and rapidly metabolized to TZR.SO and TZR.SO after oral dosing in goats. Pregnancy caused significant alterations in some of the pharmacokinetic indicators of TZR and its metabolites in goats.
\end{abstract}

Triazines, toxoplasmosis, elimination half-life, volume of distribution, HPLC

Triazines are benzene-aceto-nitrile compounds that possess antiprotozoal activity. Toltrazuril (TZR), a triazinetrione derivative, is synthetized from triazine by trimerization of nitrile (Harder and Haberkorn 1989). It is currently employed in clinical practice as a therapy for many protozoal infestations, particularly coccidiosis, neosporosis, and toxoplasmosis in various animal species (Kul et al. 2013; Qian et al. 2015; Stock et al. 2018). In addition, TZR has several favourable biological effects, for instance anti-inflammatory, analgesic, antineoplastic, and anticonvulsant activities (Harder and Haberkorn 1989).

The pharmacokinetics of TZR have been described in multiple species including rabbits (Hu et al. 2010; Kim et al. 2010), broilers (Kim et al. 2013), calves (EMEA 2000), pigs (Lim et al. 2010), ewes (Al-Qadri et al. 2020), and horses (Tobin et al. 1997). In these animals, TZR was well absorbed following oral administration and showed long plasma half-lives. Further, this compound is immediately converted to transient intermediate metabolite, toltrazuril sulphoxide (TZR.SO) and then metabolized to toltrazuril sulphone (TZR.SO $\mathrm{SO}_{2}$, which is more stable and possesses antiprotozoal activity like parent compound (Benoit et al. 1993; Mundt et al. 2007; Lim et al. 2010).

To the best of the authors' knowledge, no data exist concerning the pharmacokinetics of toltrazuril in goats, especially in pregnant ones. The physiological changes associated with gestation may affect the disposition of the administered drug (Jeffries et al. 1988;

Address for correspondence:

Dr. Sara T. Elazab

Department of Pharmacology

Faculty of Veterinary Medicine

Mansoura University, Mansoura, 35516, Egypt 
Loebstein et al. 1997). Therefore, the current study was performed with the objectives to explore the pharmacokinetic characteristics of TZR and its derivatives TZR. SO and TZR. $\mathrm{SO}_{2}$ in pregnant and non-pregnant goats after oral administration using high-performance liquid chromatography (HPLC) detection assay.

\section{Materials and Methods}

\section{Chemicals}

Toltrazuril oral suspension (Baycox ${ }^{\circledR} 5 \%$, Bayer Animal Health, KS, USA) was used in the animal experiment. The reference standards of TZR, TZR.SO, and TZR.SO, were provided by Sigma-Aldrich (St. Louis, MO, USA). The HPLC grade methanol, acetonitrile and ethyl acetate were procured from Fisher Scientific (Waltham, MA, USA). Milli-Q system (Waters Corp., Milford, MA) was utilized to purify water for HPLC analysis.

Animals and experimental design

The study was conducted using ten healthy Baladi female goats, 2-3 years old. They were obtained from a private farm in the Dakahlia Governorate, Egypt. The goats were allotted according to their pregnancy status to two groups with 5 goats each. The pregnancy status was identified using ultrasonography. The first group involved non-pregnant goats, while the second group included pregnant goats at a gestation period from 2 to 3 months. The animals were placed individually in indoor pens in the Shoha Hospital, Faculty of Veterinary Medicine, Mansoura University, Egypt. Throughout the study, they were supplied with commercial pelleted feed (Al-Mohandes Co., Tanta, Egypt) and grass hay free of any medication, and with free access to water. The commercial pelleted feed consisted of $40 \%$ wheat bran, $30 \%$ yellow corn, $24 \%$ cotton seed meal, $3 \%$ molasses, $2 \%$ limestone, and $1 \%$ sodium chloride. The animals were allowed to acclimate for 15 days before the commencing of the study. The health status of goats was evaluated through physical examination. All goats received a single oral dose of $20 \mathrm{mg} / \mathrm{kg}$ of toltrazuril (Al-Qadri et al. 2020). Then, the goats were observed daily during the study period for any potential adverse effects such as gastrointestinal disturbances or any changes in the vital signs including the pulse, temperature, and respiration. The study protocol was reviewed and accepted by the Research Ethics Committee of the Faculty of Veterinary Medicine, Mansoura University, Mansoura, Egypt (approval No. R/89).

\section{Blood sampling}

Blood samples of $2 \mathrm{ml}$ each were obtained from all goats through jugular vein puncture at time 0 (before toltrazuril administration), 0.5, 1, 2, 4, 6, 8, 12, 16, 24, 48, 72 h and 5, 7, 9, 12, 16, 20, 24, 27, 30, and 35 days post toltrazuril administration. Blood was immediately transferred to heparinized tubes and centrifuged at $1257 \times g$ for $10 \mathrm{~min}$ and harvested plasma was preserved at $-70{ }^{\circ} \mathrm{C}$ until assayed.

Analysis of toltrazuril and its metabolites in plasma samples

Sample preparation

A stock solution of TZR, TZR.SO, and TZR. $\mathrm{SO}_{2}$ pure standards in methanol was prepared at a concentration of $1 \mathrm{mg} / \mathrm{ml}$. Then, this stock solution was diluted using blank goat plasma as a diluent to prepare standard solutions of TZR, TZR.SO, and TZR.SO at concentrations of $0.05,0.1,0.25,0.5,2.5,5,25,50,100 \mu \mathrm{g} / \mathrm{ml}$.

Preparation of plasma samples and standards for HPLC analysis was performed following a previously reported technique (A1-Qadri et al. 2020) with some modifications. In brief, $100 \mu 1$ aliquot of the plasma samples were added to $1 \mathrm{ml}$ ethyl acetate and vortexed for $1 \mathrm{~min}$. Then, the mixture was centrifuged at $173 \times g$ for $10 \mathrm{~min}$. After centrifugation, the supernatant was transferred to another tube and evaporated to dryness. The residue was redissolved in $200 \mu$ l acetonitrile. The extracted sample $(100 \mu \mathrm{l})$ was injected into the HPLC column.

\section{Chromatographic conditions}

The concentrations of TZR and its metabolites in plasma were quantified according to the method announced by Zhaoling et al. (2014), with few modifications. The HPLC Agilent Series 1200 quaternary gradient pump, Series 1200 autosampler, Series 1200 UV VIS detector adjusted at $243 \mathrm{~nm}$, and HPLC 2D Chemstation software (Hewlett-Packard, Les Ulis, France) were utilized. A Phenomenex C18 column $(5 \mu \mathrm{m}, 250 \mathrm{~mm} \times 4.6 \mathrm{~mm}) \mathrm{was}$ used for chromatographic separations. The analytical assay utilized a binary-gradient mobile phase with water and acetonitrile as mobile phase $\mathrm{A}$ and $\mathrm{B}$, respectively. The gradient time program was as follows: $0-5 \mathrm{~min}$ (A-B, 48: $52 \mathrm{v} / \mathrm{v}) ; 5.1-8 \min (\mathrm{A}-\mathrm{B}, 30: 70 \mathrm{v} / \mathrm{v}) ; 8.1-12 \min (\mathrm{A}-\mathrm{B}, 0: 100 \mathrm{v} / \mathrm{v}) ; 12.1-15 \mathrm{~min}(\mathrm{~A}-\mathrm{B}, 48: 52 \mathrm{v} / \mathrm{v})$. The mobile phase was delivered at a rate of $0.8 \mathrm{ml} / \mathrm{min}$. The retention times of TZR, TZR.SO, and TZR.SO $\mathrm{SOre}_{2} .5$, 3.5 , and $4.4 \mathrm{~min}$, respectively.

The validation of the HPLC method was carried out by assessing recovery, sensitivity, precision, and linearity (Table 1). A linear correlation $\left(\mathrm{R}^{2}>0.99\right)$ was detected in the standard curve of TZR in the range of $0.05-100 \mu \mathrm{g} / \mathrm{ml}$. The lower limits of detection (LOD) and quantification (LOQ) of TZR were 0.02 and $0.05 \mu \mathrm{g} / \mathrm{ml}$, respectively. The TZR.SO calibration curve was linear through the concentrations of $0.25-100 \mu \mathrm{g} / \mathrm{ml}$. Moreover, linearity of the TZR.SO calibration curve was observed in the range of $0.1-100 \mu \mathrm{g} / \mathrm{ml}$. The LOD and LOQ were 0.08 and $0.25 \mu \mathrm{g} / \mathrm{ml}$ for TZR.SO, and 0.03 and 0.1 for TZR.SO ${ }_{2}$. 
Table 1. Validation indicators of the high-performance liquid chromatography (HPLC) assay utilized for determination of TZR, TZR.SO, and TZR. $\mathrm{SO}_{2}$. Data for recovery are presented as mean $\pm \mathrm{SEM}$.

\begin{tabular}{lccccc}
\hline Analyte & Recovery (\%) & Intra-day RSD (\%) & Inter-day RSD (\%) & LOD & LOQ \\
\hline TZR & $104.53 \pm 6.07$ & 3.34 & 3.46 & 0.02 & 0.05 \\
TZR.SO & $102.49 \pm 2.07$ & 4.30 & 4.80 & 0.08 & 0.25 \\
TZR.SO $_{2}$ & $98.12 \pm 2.08$ & 5.01 & 5.35 & 0.03 & 0.1 \\
\hline
\end{tabular}

TZR - toltrazuril; TZR.SO - toltrazuril sulphoxide; TZR.SO - toltrazuril sulphone.

Intra-day relative standard deviation (RSD) and Inter-day RSD \% for TZR, TZR.SO, and TZR.SO $(\mathrm{n}=6,0.25 \mu \mathrm{g} / \mathrm{ml})$. Mean recovery $\%$ for TZR (using spiked concentrations in the range of $0.05-100 \mu \mathrm{g} / \mathrm{ml}$ in triplicate analysis.

Mean recovery $\%$ for TZR.SO (using spiked concentrations in the range of $0.25-100 \mu \mathrm{g} / \mathrm{ml}$ in triplicate analysis.

Mean recovery $\%$ for TZR. $\mathrm{SO}_{2}$ (using spiked concentrations in the range of $0.1-100 \mu \mathrm{g} / \mathrm{ml}$ in triplicate analysis.

Pharmacokinetic analysis

Non-compartmental model (WinNonlin 8.3 software [Certara, USA]) was applied to determine the pharmacokinetic features of TZR and its metabolites in each goat as described in literature (Kim et al. 2010; Lim et al. 2010; Al-Qadri et al. 2020). The peak plasma concentration $\left(\mathrm{C}_{\max }\right)$ and the time to $\mathrm{C}_{\max }\left(\mathrm{T}_{\max }\right)$ were identified from the relationship between concentrations versus time. The linear-log trapezoidal approach was used to measure the area under the plasma concentration-time curve from time zero to the last sample $\left(\mathrm{AUC}_{0-\mathrm{last}}\right)$. The elimination half-life $\left(\mathrm{T}_{1 / 2 \lambda z}\right)$ was estimated by linear regression using the slope of the terminal data points of the semilogarthmic plasma concentration-time plots $\left(T_{1 / 2 \lambda z}=0.693 / \lambda z\right.$; where $\lambda z$ is the first order rate constant).

Statistical analysis

All data were presented as means \pm SEM. The normal distribution of data was assessed utilizing ShapiroWilk test. Differences in the plasma concentrations of TZR, TZR.SO, and TZR. $\mathrm{SO}_{2}$ between pregnant and non-pregnant goats were investigated employing the 2-way analysis of variance. Mean comparisons were conducted using Bonferroni test. The pharmacokinetic indicators of TZR and its metabolites from pregnant and non-pregnant goats were compared utilizing Wilcoxon's rank sum test. If $P<0.05$, differences were regarded as significant. These calculations were undertaken using Prism 7.0 (Graph Pad, USA).

\section{Results}

During the study period, all goats were healthy, and no obvious side effects were manifested during or after TZR therapy. The mean plasma concentrations of TZR at different time points following a single oral administration at $20 \mathrm{mg} / \mathrm{kg}$ in non-pregnant and pregnant goats are exhibited on a semilogarithmic chart in Fig. 1. TZR plasma concentrations were higher than the LOQ $(0.05 \mu \mathrm{g} / \mathrm{ml})$ up to the collected time points 24 and 27 days in non-pregnant and pregnant goats, respectively. A significant increase $(P<0.05)$ in the concentrations of TZR was detected in pregnant goats compared to non-pregnant goats at $12,16,24,48,72 \mathrm{~h}, 5,7,9$, and 12 days-post treatment.

Figure 2 shows semilogarithmic graphs of TZR.SO concentrations in non-pregnant and pregnant goats after oral administration of TZR at a single dose of $20 \mathrm{mg} / \mathrm{kg}$. TZR.SO rapidly appeared in plasma and could be detected until 12 days post TZR administration to non-pregnant goats; whereas, in pregnant goats, it was quantified $(>0.25 \mu \mathrm{g} / \mathrm{ml})$ up to 16 days. Moreover, plasma TZR.SO concentrations were significantly higher $(P<0.05)$ in pregnant goats compared to non-pregnant ones at $72 \mathrm{~h}, 5$, and 7 days after TZR oral therapy.

The plasma concentration time plots of TZR. $\mathrm{SO}_{2}$ after a single oral dose of TZR at $20 \mathrm{mg} / \mathrm{kg}$ to non-pregnant and pregnant goats are displayed in Fig. 3. No TZR.SO concentration $_{2}$ could be detected in plasma after 30 and 35 days of TZR medication in non-pregnant and pregnant goats, respectively. In the non-pregnant group, the concentrations of TZR. $\mathrm{SO}_{2}$ were significantly lower $(P<0.05)$ than in the pregnant one at 9,12 , and 16 days post TZR treatment. 


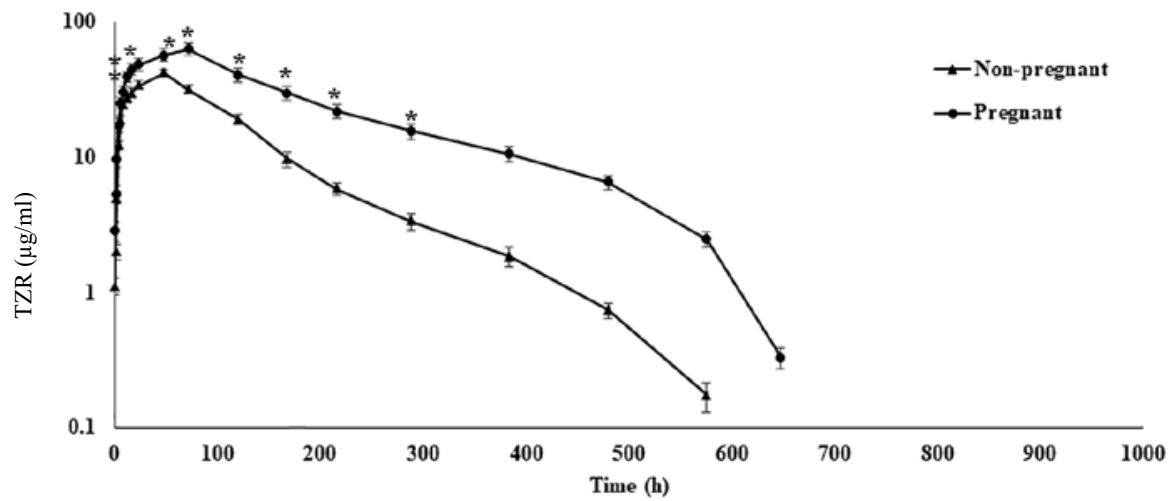

Fig. 1. Mean toltrazuril (TZR) plasma concentrations following a single oral administration in non-pregnant and pregnant goats at a dose of $20 \mathrm{mg} / \mathrm{kg}$.

Values are shown as mean $\pm \operatorname{SEM}(\mathrm{n}=5) .{ }^{*} P<0.05$

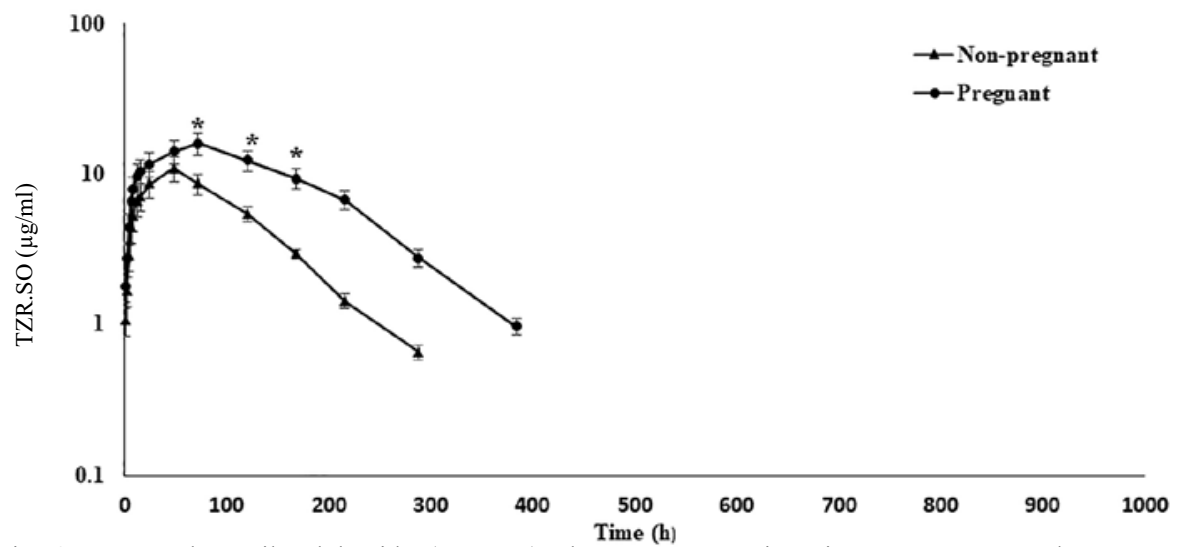

Fig. 2. Mean toltrazuril sulphoxide (TZR.SO) plasma concentrations in non-pregnant and pregnant goats following oral administration of toltrazuril (TZR) at a single dose of $20 \mathrm{mg} / \mathrm{kg}$.

Values are expressed as mean $\pm \operatorname{SEM}(\mathrm{n}=5) .{ }^{*} P<0.05$

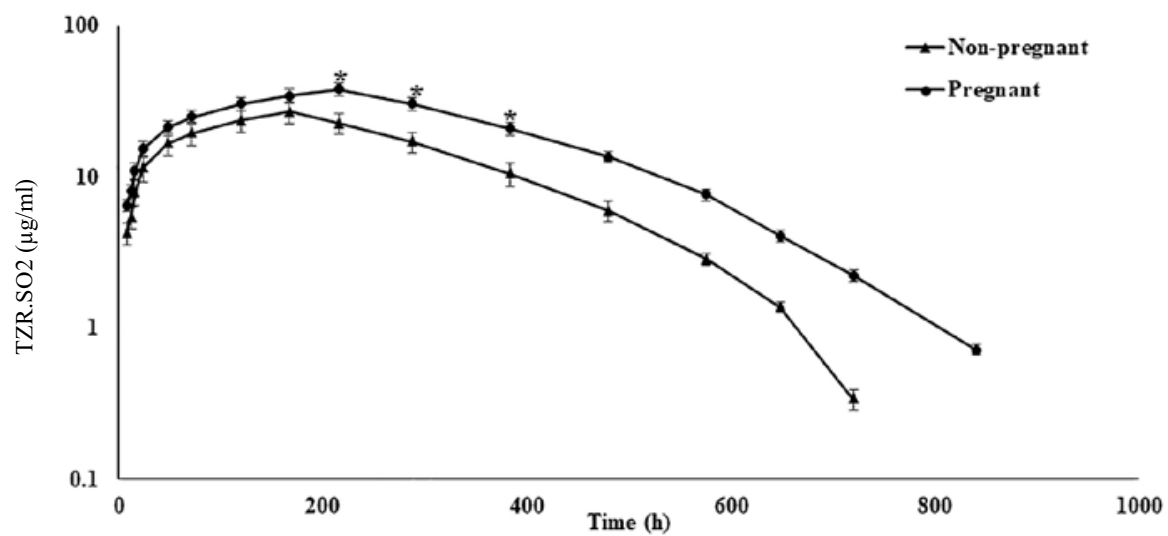

Fig. 3. Plasma concentration-time plots of toltrazuril sulphone (TZR.SO2) after administration of a single oral dose of toltrazuril (TZR) at $20 \mathrm{mg} / \mathrm{kg}$ in non-pregnant and pregnant goats.

Values are presented as mean \pm SEM $(n=5) . * P<0.05$ 
Table 2 elucidates the pharmacokinetic features of TZR in pregnant and non-pregnant goats following its oral administration once at $20 \mathrm{mg} / \mathrm{kg}$. The values of $\mathrm{C}_{\max } \mathrm{T}_{\max }$, and $\mathrm{AUC}_{\text {0-last }}$ were significantly $(P<0.05)$ higher in pregnant goats compared to non-pregnant ones. Although $\mathrm{T}_{1 / 2 \lambda z}$ and the mean residence time (MRT) were longer in pregnant animals relative to the non-pregnant ones, this difference was not significant. In addition, in pregnant goats, the volume of distribution (Vz_F_obs) and clearance (Cl_F_obs) were significantly lower $(P<0.05)$ than in the non-pregnant ones.

Table 2. Pharmacokinetic indicators of TZR after its oral administration at a single dose $(20 \mathrm{mg} / \mathrm{kg} \mathrm{BW})$ in nonpregnant and pregnant goats. Data are expressed as mean $\pm \operatorname{SEM}(n=5)$.

\begin{tabular}{lcc}
\hline Indicator & TZR in non-pregnant goats & TZR in pregnant goats \\
\hline $\mathrm{C}_{\text {max }}(\mu \mathrm{g} / \mathrm{ml})$ & $42.20 \pm 2.56$ & $63.12 \pm 7.01^{*}$ \\
$\mathrm{~T}_{\text {max }}(\mathrm{h})$ & $48.00 \pm 0.00$ & $72.00 \pm 0.00^{*}$ \\
$\lambda \mathrm{z}(1 / \mathrm{h})$ & $0.011 \pm 0.001$ & $0.010 \pm 0.002$ \\
$\mathrm{~T}_{1 / 2 \lambda z}(\mathrm{~h})$ & $64.07 \pm 3.58$ & $70.04 \pm 12.49$ \\
$\mathrm{AUC}$ & $5401.33 \pm 388.10$ & $12908.48 \pm 1480.85^{*}$ \\
$\mathrm{Vz}$ _F_obs $(\mu \mathrm{gl} / \mathrm{kg})$ & $353.05 \pm 43.09$ & $161.18 \pm 32.92^{*}$ \\
Cl_F_obs $(\mathrm{ml} / \mathrm{h} / \mathrm{kg})$ & $3.76 \pm 0.27$ & $1.62 \pm 0.17^{*}$ \\
$\mathrm{MRT}(\mathrm{h})$ & $110.73 \pm 3.72$ & $170.97 \pm 2.52$ \\
\hline
\end{tabular}

TZR - toltrazuril; $\mathrm{C}_{\max }$ - peak plasma concentration; $\mathrm{T}_{\max }$ - time to the highest plasma concentration; $\lambda \mathrm{z}$ - the first order rate constant; $\mathrm{T}_{1 / 2} \mathrm{~m}_{\mathrm{z}}$ elimination half-life; $\mathrm{AUC}_{0 \text { - last }}$ - area under the plasma concentration-time profile from 0 to last time; Vz_F_obs - volume of distribution scaled by bioavailability; Cl_F_obs - clearance divided by bioavailability; MRT - mean residence time; $* P<0.05$

The pharmacokinetic indicators of TZR.SO in non-pregnant and pregnant goats after receiving TZR once orally at $20 \mathrm{mg} / \mathrm{kg}$ are summarized in Table 3 . A significant increase $(P<0.05)$ in the values of $\mathrm{T}_{\max }$ and $\mathrm{AUC}_{\text {0-ast }}$ of TZR.SO was seen in the pregnant group compared to the non-pregnant one. Meanwhile, a significant decrease $(P<0.05)$ in values of Vz_F_obs and Cl_F_obs was noticed in pregnant goats. There were no significant differences in $\mathrm{C}_{\max }, \mathrm{T}_{1 / 2 \lambda z}$, and MRT between the two groups.

Table 3. Pharmacokinetic indicators of TZR.SO after administration of a single oral dose of TZR (20 mg/kg BW) to non-pregnant and pregnant goats. Data are expressed as mean $\pm \operatorname{SEM}(n=5)$.

\begin{tabular}{lcc}
\hline Indicator & TZR.SO in non-pregnant goats & TZR.SO in pregnant goats \\
\hline $\mathrm{C}_{\text {max }}(\mu \mathrm{g} / \mathrm{ml})$ & $10.98 \pm 2.07$ & $16.02 \pm 2.75$ \\
$\mathrm{~T}_{\text {max }}(\mathrm{h})$ & $48.00 \pm 0.00$ & $72.00 \pm 0.00^{*}$ \\
$\lambda z(1 / \mathrm{h})$ & $0.0127 \pm 0.0009$ & $0.0113 \pm 0.0005$ \\
$\mathrm{~T}_{1 / 2 \lambda z}(\mathrm{~h})$ & $55.66 \pm 3.76$ & $61.82 \pm 2.88$ \\
$\mathrm{AUC}_{\text {0-last }}(\mu \mathrm{g} * \mathrm{~h} / \mathrm{ml})$ & $1328.62 \pm 166.33$ & $2990.00 \pm 479.52^{*}$ \\
$\mathrm{VZ}_{-}$_obs $(\mathrm{ml} / \mathrm{kg})$ & $1249.36 \pm 190.79$ & $642.02 \pm 101.60^{*}$ \\
Cl_F_obs $(\mathrm{ml} / \mathrm{h} / \mathrm{kg})$ & $15.17 \pm 1.47$ & $7.20 \pm 1.15^{*}$ \\
$\mathrm{MRT}(\mathrm{h})$ & $92.41 \pm 5.67$ & $129.52 \pm 2.29$ \\
\hline
\end{tabular}

TZR - toltrazuril; TZR.SO - toltrazuril sulphoxide; $\mathrm{C}_{\max }$ - peak plasma concentration; $\mathrm{T}_{\max }$ - time to the highest plasma concentration; $\lambda z$ - the first order rate constant; $T_{1 / 2}{ }_{\lambda z}$ - elimination half-life; AUC $\max _{0 \text { last }}$ area under the plasma concentration-time profile from 0 to last time; Vz_F_obs - volume of distribution scaled by bioavailability; Cl_F_obs - clearance divided by bioavailability; MRT - mean residence time; ${ }^{*} P<0.05$ 
Table 4 illustrates the main pharmacokinetic indicators of TZR.SO obtained after a single oral dose of $20 \mathrm{mg} / \mathrm{kg}$ TZR to non-pregnant and pregnant goats. Statistical comparison of theses indicators between both groups revealed no significant difference in the $\mathrm{C}_{\max }, \mathrm{T}_{1 / 2 \lambda z}, \mathrm{MRT}$, and Vz_F_obs. Whereas, the differences in $\mathrm{T}_{\max }$ and $\mathrm{AUC}_{0 \text {-last }}$ between the two groups were significant $(P<0.05)$ as their values increased in the pregnant group. Furthermore, the Cl_F_obs was significantly lower $(P<0.05)$ in pregnant goats compared to non-pregnant ones.

Table 4. Pharmacokinetic indicators of TZR.SO $\mathrm{SO}_{2}$ after administration of a single oral dose of TZR (20 mg/kg BW) to non-pregnant and pregnant goats. Data are expressed as mean $\pm \operatorname{SEM}(n=5)$.

\begin{tabular}{lcc}
\hline Parameters & TZR. $\mathrm{SO}_{2}$ in non-pregnant goats & TZR. $\mathrm{SO}_{2}$ in pregnant goats \\
\hline $\mathrm{C}_{\text {max }}(\mu \mathrm{g} / \mathrm{ml})$ & $27.00 \pm 4.35$ & $38.10 \pm 3.94$ \\
$\mathrm{~T}_{\text {max }}(\mathrm{h})$ & $168.00 \pm 0.00$ & $216.00 \pm 0.00^{*}$ \\
$\lambda z(1 / \mathrm{h})$ & $0.0119 \pm 0.0020$ & $0.00090 \pm 0.0003$ \\
$\mathrm{~T}_{1 / 2 \lambda z}(\mathrm{~h})$ & $73.13 \pm 18.29$ & $77.42 \pm 2.82$ \\
$\mathrm{AUC}_{\text {-last }}(\mu \mathrm{g} * \mathrm{~h} / \mathrm{ml})$ & $8528.28 \pm 1355.11$ & $14257.44 \pm 1359.38^{*}$ \\
$\mathrm{Vz}_{-}$obs $(\mathrm{ml} / \mathrm{kg})$ & $252.07 \pm 64.75$ & $160.88 \pm 13.89$ \\
$\mathrm{Cl}{ }_{-}$obs $(\mathrm{ml} / \mathrm{h} / \mathrm{kg})$ & $2.58 \pm 0.39$ & $1.45 \pm 0.14^{*}$ \\
$\mathrm{MRT}(\mathrm{h})$ & $236.74 \pm 3.10$ & $276.37 \pm 3.53$ \\
\hline
\end{tabular}

TZR - toltrazuril; TZR.SO - toltrazuril sulphone; $\mathrm{C}_{\max }$ - peak plasma concentration; $\mathrm{T}_{\text {max }}$ - time to the highest plasma concentration; $\lambda \mathrm{z}$ - the first order rate constant; $\mathrm{T}_{1 / 2}{ }_{\mathrm{z}}$ - elimination half-life; $\mathrm{AUC}_{0 \text { - last - }}$ area under the plasma concentration-time profile from 0 to last time; Vz_F_obs - volume of distribution scaled by bioavailability; Cl_F_obs - clearance divided by bioavailability; MRT - mean residence time; ${ }^{*} P<0.05$

\section{Discussion}

The present research was the first one to study the pharmacokinetics of TZR in goats. The investigation of the pharmacokinetic behaviour indicated that TZR was well absorbed after oral administration in goats. This finding is in accordance with the view of Dirikolu et al. (2009) who reported that triazine-based compounds are well absorbed following oral administration due to their lipophilic nature. The $\mathrm{C}_{\max }$ of TZR in non-pregnant goats was $42.20 \mu \mathrm{g} / \mathrm{ml}$, achieved at $48.00 \mathrm{~h}\left(\mathrm{~T}_{\max }\right)$. This value was comparable to that reported for ewes and rabbits that received the same dose orally $(20 \mathrm{mg} / \mathrm{kg})(38.00$ and $39.4 \mu \mathrm{g} / \mathrm{ml}$, respectively) (Kim et al. 2010; Al-Qadri et al. 2020). On the contrary, it was higher than that found in piglets, pigs, and broiler chickens administered TZR at $20 \mathrm{mg} / \mathrm{kg}(7.50,8.18$, and $25.20 \mu \mathrm{g} / \mathrm{ml}$, respectively) (EMEA 1999; Lim et al. 2010; Kim et al. 2013), calves that received TZR orally at $15 \mathrm{mg} / \mathrm{kg}(33.41 \mu \mathrm{g} / \mathrm{ml})$ (EMEA 2000), and horses that were given TZR orally at $10 \mathrm{mg} / \mathrm{kg}(4.5 \mu \mathrm{g} / \mathrm{ml})$ (Tobin et al. 1997). Furthermore, our results showed that the $\mathrm{T}_{1 / 2 \lambda z}$ of TZR in non-pregnant goats was $64.07 \mathrm{~h}$. This $\mathrm{T}_{1 / 2 \lambda z}$ was nearly similar to that recorded in pigs $(68.9 \mathrm{~h}, 20 \mathrm{mg} / \mathrm{kg}$ TZR, Lim et al. 2010). Lower values of $\mathrm{T}_{1 / 2 \lambda z}$ were observed following oral administration of TZR in rabbits and broilers $(56.70$ and $10.70 \mathrm{~h}$, respectively) compared to those reported in our study (Kim et al. 2010, 2013). In contrast, the $\mathrm{T}_{1 / 2 \lambda z}$ found in calves and sheep (154.00 and $160.00 \mathrm{~h}$, respectively; EMEA 2000; Al-Qadri et al. 2020) were higher than that reported in our study for non-pregnant goats, advocating that TZR is more rapidly eliminated in goats compared to sheep and calves. These discrepancies may be due to species and dose regimen variations.

After absorption from the gastrointestinal tract, TZR undergoes first-pass metabolism in the liver where it is rapidly transformed to an intermediary metabolite with a short half-life, TZR.SO, which is in turn oxidized to a more active derivative, TZR. $\mathrm{SO}_{2}$. In the present 
study, the $\mathrm{T}_{\max }$ of TZR.SO was short $(48.00 \mathrm{~h})$ in non-pregnant goats that received TZR orally at $20 \mathrm{mg} / \mathrm{kg}$. This finding points out the rapid conversion of the parent compound to TZR.SO. In contrast, a noticeable retardation of the $\mathrm{C}_{\text {max }}$ of $\mathrm{TZR} \mathrm{SO}_{2}$ was recorded (the value of $\mathrm{C}_{\max }$ was $27.00 \mu \mathrm{g} / \mathrm{ml}$ attained at $168.00 \mathrm{~h}\left[\mathrm{~T}_{\max }^{\max }\right]$ ) which suggested that the oxidation of TZR.SO was slow. These results are in agreement with the EMEA report in piglets, which clarified that the $\mathrm{T}_{\max }$ of TZR.SO and TZR.SO $\mathrm{SO}_{2}$ were 48.00 and $168.00 \mathrm{~h}$, respectively, after oral administration of TZR at $20 \mathrm{mg} / \mathrm{kg}$ (EMEA 1999).

Following oral administration of TZR to non-pregnant goats, the $\mathrm{C}_{\max }$ of TZR.SO and TZR.SO ${ }_{2}$ were 10.98 and $27.00 \mu \mathrm{g} / \mathrm{ml}$, respectively. These values were consistent with the ones reported for rabbits (12.5 and $24.9 \mu \mathrm{g} / \mathrm{ml}$, respectively; Kim et al. 2010). Nevertheless, the $\mathrm{C}_{\max }$ of TZR.SO and TZR.SO in goats were higher than those reported in sheep (5.8 and $7.00 \mu \mathrm{g} / \mathrm{ml}$, respectively; Al-Q adri et al. 2020), pigs $(4.01$ and $8.74 \mu \mathrm{g} / \mathrm{ml}$, respectively; Lim et al. 2010), and piglets (3.2 and $6.2 \mu \mathrm{g} / \mathrm{ml}$, respectively; EMEA 1999) under the same conditions.

Moreover, in the current research, the goats had shorter $\mathrm{T}_{1 / 2 \lambda z}$ for TZR.SO and TZR.SO (55.66 and $73.13 \mathrm{~h}$, respectively) than those found in rabbits ( 68.8 and $82.3 \mathrm{~h}$, respectively; Kim et al. 2010) which indicated that TZR metabolites were rapidly eliminated in goats compared to rabbits. The $\mathrm{T}_{1 / 2 \lambda_{z}}$ of TZR.SO in non-pregnant goats was similar to that reported for pigs (53.20 h; Lim et al. 2010). However, the $\mathrm{T}_{1 / 2 \lambda z}$ of TZR.SO in pigs $(245.00 \mathrm{~h})$ was longer than that reported for goats in this study.

The findings of this report demonstrated that the plasma TZR concentrations at all-post treatment time points were higher in pregnant goats compared to the non-pregnant ones. The elevated plasma TZR concentrations during pregnancy accounted for the increase in $\mathrm{C}_{\max }(50 \%)$ and systemic availability (referred to as AUC; 139\%). This may reflect an increase in the absorption of TZR from the gastrointestinal lumen in pregnant animals. The effect of pregnancy on the pharmacokinetics of TZR may be attributable to changes in the haemodynamics and body fluid volumes (Mitani et al. 1987; Perez et al. 2008). Our findings were in concordance with those of A1-Qadri et al. (2020) who reported an increase in TZR bioavailability in pregnant ewes compared to non-pregnant ones.

Furthermore, pregnant animals showed lower Cl_F_obs value for TZR than the

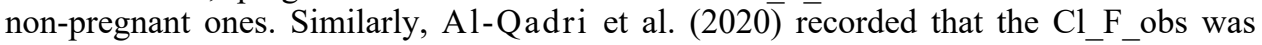
significantly decreased in pregnant ewes. This is likely due to reduced hepatic blood flow and hepatic metabolism during pregnancy (Riggs et al. 1988).

In conclusion, oral administration of TZR in goats displayed favourable pharmacokinetic characteristics with rapid absorption, high systemic availability, and extensive distribution. Furthermore, alterations in some of the pharmacokinetic indicators were observed during pregnancy.

\section{Acknowledgements}

We would like to thank the staff members of Animal Health Research institute Giza- Dokki, Egypt for their help in measuring the concentration of toltrazuril in plasma samples using HPLC. Also, we appreciate the assistance of of Mr. Ahmed Abbas with the animal experiment. We thank Mrs. Rawia Abu El-Atta, a Master degree holder in English Literature, Al-Azhar University, Cairo for the English editing.

\section{Conflict of Interests}

The authors declare no conflict of interests.

\section{References}

Al-Qadri RF, Abu-Basha EA, Ababneh MM, Bani Ismail Z, Idkaidek NM 2020: Pharmacokinetics of toltrazuril and its metabolites in pregnant and non-pregnant ewes and determination of their concentrations in milk, allantoic fluid, and newborn plasma. J Vet Pharmacol Ther 43: 339-346 
Benoit E, Buronfosse T, Moroni P, Delatour P, Riviere JL 1993: Stereoselective S-oxygenation of an aryltrifluoromethyl sulfoxide to the corresponding sulfone by rat liver cytochromes P450. Biochem Pharmacol 46: 2337-2341

Dirikolu L, Karpiesiuk W, Lehner AF, Hughes C, Granstrom DE, Tobin T 2009: Synthesis and detection of toltrazuril sulfone and its pharmacokinetics in horses following administration in dimethylsulfoxide. J Vet Pharmacol Ther 32: 368-378

European Agency for the Evaluation of European Medicines Agency (EMEA). Committee for Veterinary Products/The European Agency for the Evaluation of Medicinal Products. Toltrazuril (Extension to Pigs) Summary Report, EMEA/MRL/620/99-FINAL, 1999. Available at https://www.ema.europa.eu/en/documents/ mrl-report/toltrazuril-extension-pigs-summary-report-2-committee-veterinary-medicinal-products_en.pdf

European Agency for the evaluation of European Medicines Agency (EMEA). Committee for Veterinary Products/ The European Agency for the Evaluation of Medicinal Products. Toltrazuril (Extension to Pig) - Summary Report, EMEA/MRL/907/00-FINAL, 2000

Harder A, Haberkorn A 1989: Possible mode of action of toltrazuril: studies on two Eimeria species and mammalian and Ascaris suum enzymes. Parasitol Res 76: 8-12

Hu L, Liu C, Shang C, Yang X, Yang J 2010: Pharmacokinetics and improved bioavailability of toltrazuril after oral administration to rabbits. J Vet Pharmacol Ther 33: 503-506

Jeffrie W S, Bochner F 1988: The effect of pregnancy on drug pharmacokinetics. Med J Aust 149: 675-677

Kim MS, Lim JH, Hwang YH, Park BK, Song IB, Yun HI 2010: Plasma disposition of toltrazuril and its metabolites, toltrazuril sulfoxide and toltrazuril sulfone, in rabbits after oral administration. Vet Parasitol 169: 51-56

Kim MS, Park BK, Hwang YH, Song IB, Kim TW, Cho JH, Ham SH, Lim JH, Yun HI 2013: Pharmacokinetics and metabolism of toltrazuril and its major metabolites after oral administration in broilers. The J Poult Sci 50: $257-261$

Kul O, Yildiz K, Ocal N, Freyre A, Deniz A, Karahan S, Atmaca HT, Gokpinar S, Dincel GC, Uzunalioğlu T, Terzi OS 2013: In-vivo efficacy of toltrazuril on experimentally induced Toxoplasma gondii tissue cysts in lambs: a novel strategy for prevention of human exposure to meat-borne toxoplasmosis. Res Vet Sci 94: 269-276

Lim JH, Kim MS, Hwang YH, Song IB, Park BK, Yun HI 2010: Pharmacokinetics of toltrazuril and its metabolites, toltrazuril sulfoxide and toltrazuril sulfone, after a single oral administration to pigs. J Vet Med Sci 72: 1085-1087

Loebstein, R, Lalkin A, Koren G 1997: Pharmacokinetic changes during pregnancy and their clinical relevance. Clin Pharmacokinet 33: 328-343

Mitani, GM, Steinberg I, Lien E, Harrison C, Elkayan U 1987: The pharmacokinetics of antiarrythmic agents in pregnancy and lactation. Clin Pharmacokinet 12: 253-291

Mundt HC, Mundt-Wüstenberg S, Daugschies A, Joachim A 2007: Efficacy of various anticoccidials against experimental porcine neonatal isosporosis. Parasitol Res 100: 401-411

Perez R, Palma C, Nunez MJ, Cox J, Arboix M 2008: Pharmacokinetics of ivermectin in pregnant and nonpregnant sheep. J Vet Pharmacol Ther 31: 71-76

Qian W, Wang H, Shan D, Li B, Liu J, Liu Q 2015: Activity of several kinds of drugs against Neospora caninum. Parasitol Int 64: 597-602

Riggs KW, Axelson JE, Gruber NC, McErlane BA, McMorland, GH, Rurak DW 1988: Metoclopramide pharmacokinetics in pregnant and nonpregnant sheep. J Pharmacol Sci 77: 373-379

Stock ML, Elazab ST, Hsu WH 2018: Review of triazine antiprotozoal drugs used in veterinary medicine. J Vet Pharmacol Ther 41: 184-194

Tobin T, Dirikolu L, Harkins JD, Garnstrom DE, Carter W, Lehner F, Rees WA 1997: Preliminary pharmacokinetics of diclazuril and toltrazuril in the horse. Am Assoc Equine Pract 43: 15-16

Zhaoling J, Lifang Z, Chong Z, Xiao Z, Feiqun, X 2014: SPE-UPLC-UV method for the determination of Toltrazuril and its two metabolite residues in chicken and porcine tissues. Chromatographia 77: 1705-1712 